conditions, functional impairment, frailty, and social stressors. Rendering the homebound visible to the health care system is critical to improving care delivery and health outcomes for this vulnerable population and population health efforts. Home-based primary care (HBPC) practices have developed effective approaches to address the clinical needs of this population. Appropriate metrics are lacking to measuring the quality of care delivered by HBPC to this frail population. This session will focus on 1) understanding the characteristics of the homebound population, and; 2) elucidating efforts to address gaps in quality measurement, including the development of quality measures, a national registry, and a learning collaborative for HBPC practices.

\section{ALL IN IT TOGETHER: WHY DEVELOPMENT OF BETTER HEALTH CARE QUALITY MEASURES IS GOOD FOR CONSUMERS}

L. Walker, AARP Public Policy Institute, Washington, District of Columbia

Health care in the United States is evolving. With this change, consumers and families are taking on greater responsibilities in managing their health care - sometimes voluntarily and sometimes involuntarily. For them to navigate effectively in this new environment they and their clinicians need information about the quality of care they receive, and that information has to be meaningful to their decision-making process. This is particularly so for people who are frail, functionally impaired, and have complex chronic conditions. The homebound are a subset of this group. Consumer and patient groups, including AARP, support the development of measures in critical gap areas, such as for patients who are homebound, who would benefit tremendously from the development of home-based quality of care measures. In addition to clinical measures, consumers would welcome development of measures in domains that capture patient and caregiver experience, care coordination, safety, and quality of life.

\section{NO LONGER THE INVISIBLE HOMEBOUND: IDENTIFYING CANDIDATES FOR HOME-BASED MEDICAL CARE IN BIG DATA}

K.L. Harrison ${ }^{1,4}$, A. Altan ${ }^{2}$, S.C. Dunning 2 , C. Patterson ${ }^{2}$, C.S. Ritchie ${ }^{1}$, B.A. Leff ${ }^{3}, 1$. University of California San Francisco, School of Medicine, San Francisco, California, 2. OptumLabs, Cambridge, Massachusetts, 3. Johns Hopkins University, School of Medicine, Baltimore, Maryland, 4. San Francisco VA Medical Center, San Francisco, California

Homebound patients who could benefit from high-quality, cost-saving longitudinal home-based medical care cannot be identified easily by hospitals, health systems, or payers. Further, without a well-defined population denominator, assessments of care quality are inadequate. We addressed these gaps using the OptumLabs ${ }^{\mathrm{TM}}$ Data Warehouse, which includes more than 3 million commercial and Medicare 2014 enrollees age 65 and older. We identified two patient phenotypes who may benefit from home-based medical care: (1) patients with complex comorbid conditions already receiving home-based care ( $>2$ in-home physician visits $(\mathrm{N}=30,251)$; and (2) patients receiving substantial acute care ( $>57 \%$ with $>1$ hospitalization, ER visit, or ambulance service) and low levels of ambulatory services $(19.2 \%$ with $<2$ ambulatory visits/year); ( $\mathrm{N}=171,894)$. This project revealed a high-need patient population for whom home-based care may be beneficial and established a method for using administrative data to identify patients who are either homebound or would benefit from coordinated home-based services.

\section{SESSION 5020 (SYMPOSIUM)}

\section{BEYOND PERFORMANCE MEASURES: NOVEL INFORMATION FROM ACCELEROMETRY FOR FUNCTION}

Chair: T. Harris, NIA/Intramural Research Program, Bethesda, Maryland

Functional status has long been recognized as critical to health and independence of older persons. Self-reported and performance measures, which are quick and easy to administer, allow assessment of function and are widely used in community population studies and clinical trials. However, these measurements are limited in that they are taken at one point in time in home, clinical and laboratory settings, and thus may not be representative of a person's usual function over time. Accelerometry is an emerging technology that has gained popularity in recent years, which allows for continuous and objective assessment of daily physical activity and function over an extended period of time in the free-living environment. Although these device present new opportunities to understand functional mobility, the benefits may be limited by associated costs, methodological, and data processing challenges. This begs the question: Are the data generated from accelerometry worth the effort? In this symposium, presenters will highlight novel information derived from the accelerometers and compare with routinely available self-report or performance measures. Accelerometer types, body placement locations, processing challenges, and costs will be addressed

\section{ACTIGRAPHY FEATURES FOR PREDICTING MOBILITY DISABILITY IN OLDER ADULTS}

T.M. Manini ${ }^{1}$, M. Kheirkhahan ${ }^{1}$, C. Tudor-Locke ${ }^{2}$, N.W. Glynn ${ }^{3}$, J.M. Guralnik ${ }^{4}$, M. Pahor ${ }^{1}$, S. Ranka ${ }^{1}, 1$. University of Florida, Gainesville, Florida, 2. University of Massachusetts-Amberst, Amberst, Massachusetts, 3. University of Pittsburgh, Pittsburgh, Pennsylvania, 4. University of Maryland, Baltimore, Maryland

Actigraphy has attracted much attention for assessing and documenting physical activity in the past decade. However, there is a lack of understanding whether these data can help in detecting and/or predicting mobility function, or more specifically, mobility impairment and major mobility disability (MMD). Men ( $=357)$ and women $(\mathrm{N}=778)$ aged 70-89 years wore a tri-axial accelerometer (Actigraph GT3X) on the right hip during free-living conditions for $8.4 \pm 3.0$ days and 67 features were extracted from the accelerometer data. Sensitivity and specificity of identifying slow walkers was approximately $70 \%$ and $80 \%$, respectively. The top five features, which were related to movement pace and amount (activity counts and steps), length in activity engagement (bout length), accumulation patterns of activity, and movement variability significantly improved the prediction of MMD beyond that found with common covariates (age, 
walk speed, diseases). Actigraphy features from free-living conditions may be important for prediction of mobility phenotypes in older adults.

FRAGMENTATION OF DAILY PHYSICAL ACTIVITY: PREDICTION OF MORTALITY IN NHANES 2003-2006

J. Di, A. Leroux, J. Urbanek, A.P. Spira, J. Schrack, V. Zipunnikov, Johns Hopkins School of Public Health, Baltimore, Maryland

We review existing methods for quantifying fragmentation of accelerometry-measured physical activity and develop a unifying statistical framework that connects all methods by separately modeling switching between and duration of sedentary and active bouts. We analyzed the association of these metrics with mortality in National Health and Nutrition Examination Survey 2003-2006. We have identified that "Intradaily Variability" (1SD HR=1.32, 95\% CI 1.18-1.48), "Active Bouts Fragmentation Index" (1SD HR=1.43, 95\% CI 1.25-1.64), "Sedentary Bouts Gini Index" (1SD HR=1.15, 95\% CI 1.06-1.25), and hazard rates of distribution function of durations of active and sedentary bouts (1SD $\mathrm{HR}=1.93$, $1.40,95 \%$ CI 1.59-2.34 and 1.23-1.59) independently predict mortality after adjusting for total sedentary time, total daily activity, age, sex, education, medical comorbidities, smoking status, alcohol consumption, and mobility limitations. Future studies should concentrate on developing metrics that jointly model the frequency of switching between the sedentary and active bouts as well as the bout durations.

\section{PREDICTING VO2 MAX USING ACCELEROMETRY AND HEART RATE METRICS}

J. Schrack ${ }^{1}$, A. Leroux ${ }^{1}$, J. Fleg ${ }^{2}$, E.M. Simonsick ${ }^{3}$, V. Zipunnikov ${ }^{1}$, S.A. Studenski ${ }^{3}$, L. Ferrucci ${ }^{3}$, 1. Johns Hopkins School of Public Health, Baltimore, Maryland, 2. National Heart Lung and Blood Institute, Bethesda, Maryland, 3. National Institute on Aging, Baltimore, Maryland

Traditional physical activity metrics include reported time spent in various levels of exertion, from sedentary to vigorous. Previous research has shown an association between time spent in moderate or vigorous activities and maximal aerobic capacity (VO2 max) in older adults, but the association between accelerometry derived activity metrics and VO2 max remains undefined. We modeled the association between VO2 max and objectively measured physical activity and heart rate in 411 BLSA participants $(53 \%$ male, mean age 67 , range $31-88$ ), using percentages of heart rate reserve to define intensity of daily activities. Although the association between time spent in vigorous activities and VO2 max was significant, a continuous measure of total daily activity provided stronger results and a better model fit (accounting for $12 \%$ vs. $8 \%$ of unexplained variance), suggesting that total daily activity is a better predictor of VO2 max with aging, likely due to reduced intensity of daily activities.

\section{SEDENTARY BEHAVIOR AND PHYSICAL ACTIVITY ARE ASSOCIATED WITH PHYSICAL FITNESS: THE MAASTRICHT STUDY}

J. van der Velde ${ }^{1}$, H.H. Savelberg ${ }^{1}$, J. van der Berg ${ }^{1}$, C. Stehouwer ${ }^{2}$, P. Dagnelie ${ }^{1}$, N. Schaper ${ }^{2}$, A. Koster ${ }^{1}$, 1. Maastricht University, Maastricht, Netherlands,

\section{Maastricht University Medical Center, Maastricht,} Netherlands

We examined the associations of sedentary behavior and high intensity physical activity (HPA) with physical fitness in 1,897 men and women aged 40-75 years from The Maastricht Study. Sedentary behavior and HPA (time/ day) were measured continuously with the activPAL3 during 7 days. Physical fitness was expressed as 1) maximum power output $\left(\mathrm{W}_{\max } \mathrm{kg}^{-1}\right)$ estimated from a sub-maximal cycle ergometer test and 2) covered distance (m) from fastpaced 6 minute walk test $(6 \mathrm{MWD})$. Mean $( \pm \mathrm{SD})$ sedentary time was $9.3 \pm 1.6$ hours and median $[25 \%-75 \%]$ time in HPA was 19.8[10.1-31.1] minutes. One hour less sedentary behavior was associated with a higher $\mathrm{W}_{\max } \mathrm{kg}^{-1}(\mathrm{~B}=0.022)$ and longer 6MWD $(\mathrm{B}=2.455)$ independent of HPA $(\mathrm{p}<0.05)$. An additional 10 minutes of HPA was associated with higher $\mathrm{W}_{\text {max }} \mathrm{kg}^{-1}(\mathrm{~B}=0.036)$ and longer $6 \mathrm{MWD}(\mathrm{B}=3.088)$, independent of sedentary behavior $(p<0.01)$. HPA seems to be more important for physical fitness than sedentary behavior.

\section{USING ACCELEROMETRY TO TRACK CLINICAL TRAJECTORIES: AORTIC VALVE REPLACEMENT AS AN EXAMPLE}

T. Harris' 1 P. Green ${ }^{2}$, A. Eloyan ${ }^{3}$, V. Zipunnikov ${ }^{4}$, M. Maurer ${ }^{2}$, M. Hung ${ }^{1}$, C. Crainiceanu ${ }^{4}$, 1. Laboratory of Epidemiology and Population Sciences, NIA/Intramural Research Program, Bethesda, Maryland, 2. Columbia University College of Medicine, New York, New York, 3. Brown University School of Public Health, Providence, Rhode Island, 4. Johns Hopkins School of Public Health, Baltimore, Maryland

Accelerometry technology has been used to capture motion in studies of physical activity, where these instruments have been used to assess the total amount of activity and its intensity. Clinicians have begun to realize that these characteristics could provide information about underlying health status and the use of monitors to predict clinical outcomes has increased. This presentation will review current applications of accelerometers in clinical practice. Using data from a pilot study of 53 patients undergoing aortic valve replacement either through open heart surgery or through transcatheter valve replacement, we will show the pre-intervention accelerometry data. We will then compare postintervention accelerometry data over time, illustrating where there are significant differences between the types of valve replacement and between the pre- and the post-intervention accelerometry data.

Accelerometry technology has been used to capture motion in studies of physical activity, where these instruments have been used to assess the total amount of activity and its intensity. Among other characteristics analyzed from the raw accelerometry signal include time spent sedentary, time in sleep, duration of bouts of motion, and time walking. Clinicians have begun to realize that these characteristics could provide information about underlying health status and the use of monitors to predict clinical outcomes has increased. This presentation will review current applications of accelerometers in clinical practice. Using data from a pilot study of 53 patients undergoing aortic valve replacement either through open heart surgery or through transcatheter valve replacement, we will show the pre-intervention 